[On Penetrative Convection in the Isothermally Incompressible Fluid Approximation] // Izv. RAN, ser.: MZhG [RAS Bulletin, Series Mechanics of Liquid and Gas].-1996. - № 2. - P.40-52 (in Russian).

5. Tankin R., Farhadieh R. Effects of thermal convection currents on formation of ice // Int. J. Heat Mass Transfer. - 1971. - V.14. - P. 953-960.

6. Kuznetsova D.V., Sibgatullin I.N. Perehodnye rezhimy $\mathrm{v}$ pronikajushhej konvekcii $\mathrm{v}$ ploskom sloe. [Transitional Regimes of Penetrative Convection in a Plain Layer.] // Vestnik Nizhegorodskogo universiteta im. N.I. Lobachevskogo [Bulletin of Lobachevskiy State University of Nizhny Novgorod].- 2011.- № 4(3).- P.907-909 (in Russian).

7. $\mathrm{Hu}$ Yu-Peng, Li You-Rong, Li Ming-Hai, Zhang Li, Li Si-Zhong. Effects of enclosure geometry and thermal boundary condition on Rayleigh-Benard convection of cold water near its maximum density // Int. J. Therm. Sci. - 2017. - V.120. - P.220-232.

8. Palymskiy I.B. About convection stability of the gas-vapor mixture at the temperature close to critical // J. App. Mech. Engineer. - V. 7. - P. 53-54.

9. Goldstein R.J., Graham D.J. Stability of a horizontal fluid with zero shear boundaries // Phys. Fluids. - 1969. - V.12. - N.6. - P.1133-1137.

10. Palymskiy I.B., Fomin P.A., Hieronymus H. Rayleigh-Benard convection in a chemical equilibrium gas (simulation of surface detonation wave initiation) // App. Math. Modelling. - 2008. - V. 32. - Is. 5. - P. 660676.

\title{
APPLICATION OF THE THEORY OF OPTIMAL SET PARTITIONING BEFORE BUILDING MULTIPLICATIVELY WEIGHTED VORONOI DIAGRAM WITH FUZZY PARAMETERS
}

\author{
DOI: $10.31618 /$ ESU.2413-9335.2020.6.71.615 \\ Kiseleva E.M., \\ Prytomanova O.M., \\ Padalko V.H. \\ Oles Honchar Dnipro National University
}

\begin{abstract}
ABSTARCT
An algorithm for constructing a multiplicatively weighted Voronoi diagram involving fuzzy parameters with the optimal location of a finite number of generator points in a limited set of $n$-dimensional Euclidean space $E_{n}$ has been suggested in the paper. The algorithm has been developed based on the synthesis of methods of solving the problems of optimal set partitioning theory involving neurofuzzy technologies modifications of N.Z. Shor $r$-algorithm for solving nonsmooth optimization problems.

Keywords: multiplicatively weighted Voronoi diagram, optimal set partitioning problem, optimal location of generator points, neurofuzzy technologies, N.Z. Shor $r$-algorithm, nonsmooth optimization problems.

Introduction. Currently, hundreds of literary sources on Voronoi diagrams and their application in various fields [1]. Voronoi diagrams for two- and threedimensional spaces are used in many different areas of applied sciences. Quite apart from the fact that a large amount of known algorithms for constructing Voronoi diagrams of given finite $M$ sets of a plane (space) points, called generator points, have $O(|M| \log (|M|))$ complexity, all these algorithms are quite complicated. In addition, in practical problems the parameters of the Voronoi diagrams often may be fuzzy.

In the papers $[2,3]$, the algorithms for constructing a standard (classical) Voronoi diagram with deterministic parameters and various generalizations are suggested, based on the application of methods of optimal set partition (OSP) theory and having several advantages over the known ones, which are described in the scientific literature $[1,4,5]$. Particularly, they do not depend on the $E_{n}$ space dimension containing a limited set to be partitioned, which is independent of the geometry of the sets to be partitioned; the complexity of algorithms for plotting Voronoi diagrams based on the approach described above does not increase with the growing number of generator points; they can be used to construct not only Voronoi diagrams of a given number of generator points of fixed locations, but also with the optimal location of these

points in a limited amount of $E_{n}$ space, and other advantages. The result of such a versatile approach is the ability to easily construct not only already known Voronoi diagrams but also the new ones.

The versatility of the approach proposed in studies $[2,3]$ in the construction of Voronoi diagrams is further supported by the fact that models and methods for solving continuous problems of optimal set partitioning can be generalized in the case of fuzzy initial parameters of the problem or a requirement of a fuzzy set partitioning, resulting in a fuzzy nature of Voronoi diagrams.

In this paper, there has been developed the algorithm of constructing a multiplicatively weighted Voronoi diagram involving fuzzy parameters with optimal positioning of $N$ finite number of generator points in $\Omega$ limited set in $n$-dimensional $E_{n}(n \geq 2)$ Euclidean space. The algorithm has been developed based on the synthesis of methods of solving problems of the theory of OSP [7]) with neuro-fuzzy technologies [8]) and modifications of N.Z. Shor $r$-algorithm for solving non-smooth optimization problems $[9,10]$.

Problem setting. A classical Voronoi diagram of a finite set $M=\left\{\tau_{1}, \tau_{2}, \ldots, \tau_{N}\right\} \subset E_{n}$ of generator points $\tau_{i}=\left(\tau_{i}^{(1)}, \tau_{i}^{(2)}, \ldots, \tau_{i}^{(n)}\right), i=1,2, \ldots, N$ in $n$ dimensional Euclidean space $E_{n}(n \geq 2)$ is Voronoi polyhedra set
\end{abstract}




$$
\operatorname{Vor}\left(\tau_{i}\right)=\left\{x \in \mathrm{E}_{n}: c\left(x, \tau_{i}\right) \leq c\left(x, \tau_{j}\right), j=1,2, \ldots, N, j \neq i\right\}, i=1,2, \ldots, N
$$

of seed points $\tau_{1}, \tau_{2}, \ldots, \tau_{N}$, where $c_{i}\left(x, \tau_{i}\right)$ are In a multiplicatively weighted Voronoi diagram functions of the distance between $x$ and $\tau_{i}$ points which of the set $M=\left\{\tau_{1}, \tau_{2}, \ldots, \tau_{N}\right\} \subset E_{n}$ are defined in $E_{n}$ as a Euclidean metric.

$$
M W \operatorname{Vor}(M)=\bigcup_{\tau_{i} \in M} M W \operatorname{Vor}\left(\tau_{i}\right)
$$

each Voronoi polyhedron

$$
M W \operatorname{Vor}\left(\tau_{i}\right)=\left\{x \in E_{n}: \mathrm{c}\left(x, \tau_{i}\right) / w_{i} \leq \mathrm{c}\left(x, \tau_{j}\right) / w_{j}, j=1,2, \ldots, N, j \neq i\right\}, i=1,2, \ldots, N,
$$

represents a set of space, the weighted distance from which to the generator point $\tau_{i} \in M$ does not exceed the weighted distance to any other seed point ( $w_{i}>0, i=1,2, \ldots, N$, are given weight coefficients).

Growing crystals is one of the illustrative ways of obtaining a multiplicatively weighted Voronoi diagram [6]. When all crystals begin to grow at the same time but at different speeds, each $\tau_{i} \in M$ point gets a weight coefficient, and when measuring the distance to it, one needs to multiply the function that sets the distance by that weight coefficient.

Voronoi diagrams with fuzzy parameters appear, for example, when the weight coefficients of the two-point distance functions that determine the elements of the Voronoi diagram are fuzzy. Let us set for each $c_{i}\left(x, \tau_{i}\right)$ function of (1) a fuzzy weight $\widetilde{w}_{i}$, which depends on external factors, which may also be fuzzy, and at the same time the type of this dependency is unknown in advance.

Then (1) shall be put down as follows

$$
M W \operatorname{Vor}\left(\tau_{i}\right)=\left\{x \in E_{n}: \mathrm{c}\left(x, \tau_{i}\right) / \widetilde{w}_{i} \leq \mathrm{c}\left(x, \tau_{j}\right) / \widetilde{w}_{j}, j=1,2, \ldots, N, j \neq i\right\}, i=1,2, \ldots, N
$$

For a mathematical formulation of a problem of constructing a multiplicatively weighted Voronoi diagram with an optimal positioning of a finite number $N$ of generator points in $\Omega$ limited set of $n$ dimensional Euclidean space $E_{n}(n \geq 2)$ on the basis of methods for solving OSP problems, let us eliminate the fuzziness in (2) by the neurolinguistic identification method of unknown complex nonlinear relationship of [8].

To apply the specified method in recovering values of fuzzy parameters $\widetilde{w}_{1}, \ldots, \widetilde{w}_{N}$, without limiting the generalization of considerations, let us denote their restored values as $w$ and consider the functional

$$
\begin{gathered}
w=\frac{\sum_{k=1}^{L} d_{k} \cdot \mu * D_{k}(w)}{\sum_{k=1}^{L} \mu * D_{k}(w)}, \\
\mu_{D_{k}}^{*}(w)=\left\{\begin{array}{c}
\sum_{j=1}^{s_{k}} p_{j}^{* k}\left(y_{1}, y_{2}, \ldots, y_{q}\right), \text { if } \sum_{j=1}^{s_{k}} p_{j}^{* k}\left(y_{1}, y_{2}, \ldots, y_{q}\right) \leq 1, \\
\text { otherwise, } \\
p *_{j}^{k}\left(y_{1}, y_{2}, \ldots, y_{q}\right)=v *_{j}^{k} \prod_{i=1}^{q} \mu *_{i j}^{k}\left(y_{i}\right), \\
1+\left(\frac{y_{i}-b *_{i j}^{k}}{e *_{i j}^{k}}\right)
\end{array}, i=1, \ldots, q, j=1, \ldots, s_{k}, \mathrm{k}=1,2, \ldots, \mathrm{L},\right.
\end{gathered}
$$

where in formulas (4) - (7):

- $\mu *_{D_{k}}(w)$ is the membership function of the original variable $w$ of $D_{k}$ class, $k=1,2, \ldots, L, L$ is the number of classes (linguistic terms) of the original variable $w, d_{k}$ is the centre of the class $D_{k}$; 
- $p *_{j}^{k}\left(y_{1}, y_{2}, \ldots, y_{q}\right)$ are fuzzy production rules derived from expert and experimental information on dependency (6), $j$ is the rule number in $k$-class, $j=$ $1,2, \ldots, s_{k}, s_{k}$ - means the amount of rules in $k$-class; $v *_{j}^{k}$ - weight of $j$-rule in $k$-class of output $w$;

- $\mu *_{i j}^{k}\left(y_{i}\right)$ is the bell-shaped function of $y_{i}$ variable membership in its linguistic term in $j$-rule of $k$-class of the output variable $w, b *_{i j}^{k}$ is the coordinate of the maximum and $e *_{i j}^{k}$ is the concentration coefficient of this membership function.

It should be noted that the value $v *_{j}^{k}$ - of the weights of the rules in (6) and the parameters $t *_{i j}^{k}, e *_{i j}^{k}$ of the membership function (7) are marked by an asterisk as optimal ones, that is, the values obtained as a result of the parametric identification of the method of neurolinguistic identification, for which the deviation of the experimental data from the artificial data obtained after adjusting the fuzzy model of the object (3) reaches its minimum. Values of $\mu *_{D_{k}}(w)$, $p *_{j}^{k}\left(y_{1}, y_{2}, \ldots, y_{q}\right)$ and $\mu *_{i j}^{k}\left(y_{i}\right)$ in (4) - (7) are calculated at optimal values of $v *_{j}^{k}, b *_{i j}^{k}, e *_{i j}^{k}$.
Thus, after restoring fuzzy parameter values $\widetilde{w}_{1}, \ldots, \widetilde{w}_{N}$ in (2) using the described method of neurolinguistic identification, we obtain their recovered (crisp) values of $w_{1}, \ldots, w_{N}$.

We now formulate the mathematical setting of a problem of constructing a multiplicatively weighted Voronoi diagram with the optimal placement of a finite number of $N$ generator points in $\Omega$ limited set of $n$ dimensional Euclidean space $E_{n}(n \geq 2)$ on the basis of methods of solving OSP problems and restored values of its fuzzy parameters.

Let us assume that $\Omega$ is a given bounded set of $E_{n}$, $\tau_{1}, \tau_{2}, \ldots, \tau_{N}$ is a finite set of generator points in $\Omega$. In cases where the location of points $\tau_{1}, \tau_{2}, \ldots, \tau_{N}$ in $\Omega$ is unknown and these must be placed (selected) in $\Omega$, another Voronoi diagram can be introduced on a set $\Omega \subset E_{n}$, that is, the Voronoi diagram of points optimally located in a finite set.

We will consider the following total of Voronoi polyhedra as a multiplicatively weighted Voronoi diagram of a finite number $N$ of generator points $\tau_{1}, \tau_{2}, \ldots, \tau_{N}$ in $\Omega \subset E_{n}$ limited set

$$
M W \operatorname{Vor}\left(\tau_{i}\right)=\left\{x \in E_{n}: \mathrm{c}\left(x, \tau_{i}\right) / w_{i} \leq \mathrm{c}\left(x, \tau_{j}\right) / w_{j}, j=1,2, \ldots, N, j \neq i\right\}, i=1,2, \ldots, N
$$

of $\tau_{1}, \tau_{2}, \ldots, \tau_{N}$ points optimally placed in a finite set, for which the total weighted distance from the points of $\Omega$ set to the corresponding $\tau_{1}, \tau_{2}, \ldots, \tau_{N}$ generator points is the smallest, that is, the functional

$$
J\left(\left\{\tau_{1}, \ldots, \tau_{N}\right\}\right)=\sum_{i=1}^{N} \int_{\operatorname{Vor}\left(\tau_{i}\right)}\left(c\left(x, \tau_{i}\right) / w_{i}\right) d x
$$

takes on the minimum value

The method of solving the problem. Let's develop an approach to constructing a multiplicatively weighted Voronoi diagram of a finite number of generator points $\tau_{1}, \tau_{2}, \ldots, \tau_{N}$, optimally placed in $\Omega \subset$ $E_{n}$ finite set, with fuzzy parameters, based on the application of the mathematical apparatus of neurofuzzy technologies [8] and the OSP theory [7]. To do this, we must first formulate the corresponding continuous problem of optimal partitioning of the set of
$\mathrm{E}_{n}$ to subsets with unknown coordinates of some points characteristic of each subset, which are called the centres of subsets, being a generalization of the problem of [7].

Let $\Omega$ be a bounded Lebesgue set in $n$-dimensional Euclidean space $E_{n}$. Let a total of Lebesgue measurable subsets $\Omega_{1}, \ldots, \Omega_{N}$ of $\Omega \subset E_{n}$ be called the possible division of the $\Omega$ set into its non-intersecting subsets $\Omega_{1}, \ldots, \Omega_{N}$ if

$$
\bigcup_{i=1}^{N} \Omega_{i}=\Omega, \operatorname{mes}\left(\Omega_{i} \cap \Omega_{j}\right)=0, \quad i, j=1,2, \ldots, N(i \neq j),
$$

where mes $(\cdot)$ means Lebesgue measure.

Let $\sum_{\Omega}^{N}$ denote the class of all possible partitions of $\Omega$ set into non-intersecting subsets, $\Omega_{1}, \ldots, \Omega_{N}$ that is

$$
\sum_{\Omega}^{N}=\left\{\left(\Omega_{1}, \ldots, \Omega_{N}\right): \bigcup_{i=1}^{N} \Omega_{i}=\Omega, \operatorname{mes}\left(\Omega_{i} \cap \Omega_{j}\right)=0, \quad i, j=1,2, \ldots, N(i \neq j)\right\} .
$$

Let's introduce the functional

$$
F\left(\left\{\Omega_{1}, \ldots, \Omega_{N}\right\},\left\{\tau_{1}, \ldots, \tau_{N}\right\}\right)=\sum_{i=1}^{N} \int_{\Omega_{i}}\left(c\left(x, \tau_{i}\right) / w_{i}\right) d x
$$

where $c\left(x, \tau_{i}\right)$ is a given real-valued limited $\Omega \times \Omega$ function measured by $x=\left(x^{(1)}, \ldots, x^{(n)}\right) \in \Omega$ for any fixed point $\tau_{i}=\left(\tau_{i}^{(1)}, \ldots, \tau_{i}^{(n)}\right) \in \Omega$ for all $i=$ $1,2, \ldots, N ; w_{i}>0(i=1,2, \ldots, N)$ are given weight coefficients.

Here, integrals are thought of as being Lebesgue. We assume that a measure of a set of the limiting points of the set $\Omega_{i}, i=1, \ldots, N$ is zero.

$$
\begin{aligned}
& \text { Problem } \\
& \min _{\left\{\Omega_{1}, \ldots, \Omega_{N}\right\} \in \Sigma_{\Omega}^{N},} F\left(\left\{\Omega_{1}, \ldots, \Omega_{N}\right\},\left\{\tau_{1}, \ldots, \tau_{N}\right\}\right), \\
& \left\{\tau_{1}, \ldots, \tau_{N}\right\} \in \Omega^{N} \\
& \text { where functional } F\left(\left\{\Omega_{1}, \ldots, \Omega_{N}\right\},\left\{\tau_{1}, \ldots, \tau_{N}\right\}\right) \text { is } \\
& \text { represented in the form of }(11) ; \text { the } \tau_{i}^{(1)}, \ldots, \tau_{i}^{(n)} \\
& \text { coordinates of centres of } \tau_{i}=\left(\tau_{i}^{(1)}, \ldots, \tau_{i}^{(n)}\right) \in \Omega_{i}, i=
\end{aligned}
$$


$1,2, \ldots, N$, are unknown in advance and need to be identified.

The $\left(\left\{\Omega_{1}^{*}, \ldots, \Omega_{N}^{*}\right\},\left\{\tau_{1}^{*}, \ldots, \tau_{N}^{*}\right\}\right)$ pair which represents the minimum of functional (11) on $\Sigma_{\Omega}^{N} \times \Omega^{N}$ set may be called an optimal solution of the Problem A. In this case, let us call $\left\{\Omega_{1}^{*}, \ldots, \Omega_{N}^{*}\right\} \in \Sigma_{\Omega}^{N}$ partition an optimal partitioning of $\Omega \subset E_{n}$ set into $N$ subsets, and $\tau^{*}=\left(\tau_{1}^{*}, \ldots, \tau_{N}^{*}\right) \in \Omega^{N}$ total of $\tau_{i}^{*} \in \Omega_{i}^{*}, i=1,2, \ldots, N$ centres - the optimal centres of $\Omega_{i}^{*}$ subsets in problem A.

Problem B. Find
To solve the Problem A for each fixed $w_{i}(i=1,2, \ldots, N)$ we introduce the characteristic functions of subsets $\Omega_{i}$ :

$$
\lambda_{i}(x)=\left\{\begin{array}{l}
1, x \in \Omega_{i}, \\
0, x \in \Omega \backslash \Omega_{i}, \quad i=1, \ldots, N,
\end{array}\right.
$$

and restate the Problem $A$ in terms of characteristic functions as follows.

$$
\min _{(\lambda(\cdot), \tau) \in \Gamma \times \Omega^{\mathrm{N}}} \int_{\Omega} \sum_{i=1}^{N}\left(c\left(x, \tau_{i}\right) / w_{i}\right) \lambda_{i}(x) d x,
$$

where

$$
\begin{aligned}
& \Gamma=\left\{\lambda(x)=\left(\lambda_{1}(x), \ldots, \lambda_{N}(x)\right): \sum_{i=1}^{N} \lambda_{i}(x)=1 \text { almost everywhere (a.e.) for } x \in \Omega\right. \\
& \left.\lambda_{i}(x)=0 \vee 1 \text { a.e. for } x \in \Omega i=1, \ldots, N\right\} ; \tau=\tau_{1}, \ldots, \tau_{N} \in \underbrace{\Omega \times \ldots \times \Omega}_{N}=\Omega^{N}
\end{aligned}
$$

For Problem B, in [7], we prove the following theorem 1, which establishes the optimal solution $\left(\lambda_{*}(\cdot), \tau_{*}\right)$.

Theorem 1. Components of a characteristic vector function $\quad \lambda_{*}(x)=$ $\left(\lambda_{* 1}(x), \ldots, \lambda_{* i}(x), \ldots, \lambda_{* N}(x)\right)$ that matches the

optimal solution $\left(\Omega_{* 1}, \ldots, \Omega_{* i}, \ldots, \Omega_{* N}\right)$ of the Problem $\mathbf{A}$ for $i=1, \ldots, N$ and almost all $x \in \Omega$ are as follows:

$$
\lambda_{*_{i}}(x)=\left\{\begin{aligned}
1, & \text { if } c\left(x, \tau_{*_{i}}\right) / w_{i} \leq c\left(x, \tau_{*_{j}}\right) / w_{j}, \\
& i \neq j \text { a.e. for } x \in \Omega, j=1, \ldots, N, \text { then } x \in \Omega_{*_{i}} \\
0 & \text { otherwise, }
\end{aligned}\right.
$$

the optimal solution to the problem, which is dual to problem $\mathrm{B}$, is chosen as $\tau_{* 1}, \ldots, \tau_{* N}$ :

$$
G(\tau)=\int_{\Omega} \min _{i=1, \ldots, N}\left[c\left(x, \tau_{i}\right) / w_{i}\right] d x \rightarrow \min , \tau \in \Omega^{N} .
$$

We now present Theorem 2, based on the results of $[7,11]$ papers, which summarizes our considerations and will be further used in the formulation of the algorithm for solving Problem A.

Theorem 2. Components of a characteristic vector function $\lambda_{*}(x)=\left(\lambda_{* 1}(x), \ldots, \lambda_{* i}(x), \ldots, \lambda_{* N}(x)\right)$ that

$$
\lambda_{*_{i}}(x)=\left\{\begin{aligned}
1, & \text { if } c\left(x, \tau_{*_{i}}\right) / w_{i} \leq c\left(x, \tau_{*_{j}}\right) / w_{j}, \\
& i \neq j \text { a.e. for } x \in \Omega, j=1, \ldots, N, \text { then } x \in \Omega_{*_{i}}, \\
0 & \text { otherwise, }
\end{aligned}\right.
$$

the optimal solution of the problem is chosen as $\tau_{* 1}, \ldots, \tau_{* N}$

$$
G(\tau)=\int_{\Omega} \min _{i=1, \ldots, N}\left[c\left(x, \tau_{i}\right) / w_{i}\right] d x \rightarrow \min , \tau \in \Omega^{N} .
$$

Here, each parameter $w_{i}, i=1, \ldots, N$ previously specified as the output $w$ that depends on $y_{1}, \ldots, y_{q}$ inputs as $w=w\left(y_{1}, \ldots, y_{q}\right)$, is calculated by formulas (4) - (7).

Problem A solution algorithm. To describe the algorithm, we define $i$-component, $i=1, \ldots, N$, being 
the component of the generalized gradient vector of $g_{G}^{\tau}(\tau)=\left(g_{G}^{\tau_{1}}(\tau), \ldots, g_{G}^{\tau_{i}}(\tau), \ldots, g_{G}^{\tau_{N}}(\tau)\right) \quad$ functions

(13) at $\tau$ point as follows:

$$
g_{G}^{\tau_{i}}(\tau)=\int_{\Omega} g_{c}^{\tau_{i}}(x ; \tau) \lambda_{i}(x) d x, \quad i=1, \ldots, N
$$

where $g_{c}^{\tau_{i}}(x, \tau)$ - $i$-component of $N$-dimensional vector of generalized gradient $g_{c}^{\tau}(x, \tau)$ of $c\left(x, \tau_{i}\right)$ In the formula (14) $\lambda_{i}(x), i=1, \ldots, N$, is defined as follows:

$$
\lambda_{i}(x)=\left\{\begin{array}{l}
1, \text { if } c\left(x, \tau_{i}\right) / w_{i} \leq c\left(x, \tau_{j}\right) / w_{j}, \\
\quad \text { in other cases, } \underset{J}{\text { ien }} 1, \ldots, N, \text { then } x \in \Omega_{i} \\
0 \text { otherwise, }
\end{array}\right.
$$

where $w_{i}, i=1, \ldots, N$, are calculated by formulas (4) - (7).

Algorithm. We place $\Omega$ domain to $n$-dimensional parallelepiped $\mathrm{P}$, the sides of which are parallel to the axes of the Cartesian coordinate system. We cover the parallelepiped $\mathrm{P}$ with a rectangular grid and set the initial approximation $\tau=\tau^{(0)}$. We calculate $\lambda^{(0)}(x)$ value at the nodes of the grid according to formulas (12), taking into account (4) - (7) formulas for calculating the parameters $w_{i}$, at $\tau=\tau^{(0)} ; g_{G}(\tau)$ value - by the formula (14) at $\lambda(x)=\lambda^{(0)}(x), \tau=\tau^{(0)}$; select the initial test step of $r$-algorithm $h_{0}>0$ and find

$$
\tau^{1}=P_{\Pi}\left(\tau^{0}-h_{0} \frac{H_{1} g_{G}\left(\tau^{0}\right)}{\sqrt{\left(H_{1} g_{G}\left(\tau^{0}\right), g_{G}\left(\tau^{0}\right)\right)}}\right),
$$

$\mathrm{P}_{\Pi}$ is a projection operator on $\Pi$.

Proceed to the second step.

Let the result of calculations after $k(k=1,2, \ldots$,$) steps of the algorithm be the obtained$ values of $\tau^{(k)}, \lambda^{(k-1)}(x)$ in the nodes of the grid.

Let us describe $(k+1)$ step.

1. We calculate $\lambda^{(k)}(x)$ value at the nodes of the grid according to formulas (15), taking into account (4) - (7) formulas to calculate the parameters of $w_{i}$, at $\tau=\tau^{(k)}$.

2. We find $g_{G}(\tau)$ value by formulas (14) at $\lambda(x)=\lambda^{(k)}(x), \tau=\tau^{(k)}$.

3. We then take $(k+1)$ step of r-algorithm in $N$ form, an iterative formula of which is as follows

$$
\tau^{k+1}=P_{\Pi}\left(\tau^{k}-h_{k} \frac{H_{k+1} g_{G}\left(\tau^{k}\right)}{\sqrt{\left(H_{k+1} g_{G}\left(\tau^{k}\right), g_{G}\left(\tau^{k}\right)\right)}}\right)
$$

4. If the condition $\left\|\tau^{k}-\tau^{k+1}\right\| \leq \varepsilon, \varepsilon>0$,

(16) is not met, we go to $(k+2)$ step of the algorithm, otherwise - to cl. 5 .

5. Let $\lambda_{*}(x)=\lambda^{(l)}(x), \tau_{*}=\tau^{(l)}$, where $l$ is the iteration number at which condition (16) is satisfied.

6. We calculate the optimal value of the objective function $G(\tau)$ of (13) by the formula

$$
G(\tau)=\int_{\Omega} \min _{i=1, \ldots, N}\left[c\left(x, \tau_{i}\right) / w_{i}\right] d x,
$$

at $\tau=\tau_{*}$ and $w_{i}, i=1, \ldots, N$ calculated by formulas (14) - (17).

The described algorithm.

Thus, as a result of the solution of Problem A by the algorithm described above, which is based on theorem 2 above, we obtain a total of Voronoi polyhedra (3) of generator points $\boldsymbol{\tau}_{\boldsymbol{i}}, i=1, \ldots, N$ :

$$
\operatorname{Vor}\left(\tau_{i}\right)=\left\{x \in \Omega \subset E_{n}: c\left(x, \tau_{i}\right) / w_{i} \leq c\left(x, \tau_{j}\right) / w_{j}, i \neq j, j=1, \ldots, N\right\},
$$

but unlike the standard Voronoi diagram (1), where points $\tau_{1}, \ldots, \tau_{N}$ are fixed and parameters $w_{i}, i=1, \ldots, N$ are clear, in order to find the coordinates of the generator points $\tau_{1}, \ldots, \tau_{N}$, optimally

$$
G(\tau)=\int_{\Omega} \min _{i=1, \ldots, N}\left[c\left(x, \tau_{i}\right) / w_{i}\right] d x \rightarrow \min , \tau \in \Omega^{N}=\underbrace{\Omega \times \ldots \times \Omega}_{N},
$$


with a non-differentiated objective function $G(\tau)$ and $w_{i}, i=1, \ldots, N$ parameters recovered using the neurolinguistic identification method of unknown complex nonlinear relationship.

Summary. The method and algorithm has been suggested for constructing a multiplicatively weighted Voronoi diagram involving fuzzy parameters with optimal location of a finite number of generator points in a limited set of $n$-dimensional Euclidean space $E_{n}$. The method is based on the formulation of an appropriate continuous problem of optimal set partitioning into non-intersecting subsets, where the centers of these subsets are located involving fuzzy parameters in the objective functional and with the criterion of the partition quality, which provides an appropriate Voronoi diagram with fuzzy parameters. The method of solving the above problem of optimal set partitioning is based on the application of the mathematical apparatus developed in [11], while the method of neurolinguistic identification, developed in [8], was used to eliminate the fuzziness in the OSP problem.

\section{Bibliographic References}

1.Preparata F., Sheimos M. Computational geometry: an introduction. Springer. First Edition edition, 1993.390 p.

2.Kiseleva E.M., Koriashkina L.S. Theory of continuous optimal set partitioning problems as a universal mathematical formalism for constructing Voronoi diagrams and their generalizations I. Theoretical foundations // Cybernetics and Systems Analysis, vol. 51, № 3, pp. 325-335 (2015). DOI 10.1007/s10559-015-9725-x.

3.Kiseleva E.M., Koriashkina L.S. Theory of continuous optimal set partitioning problems as a universal mathematical formalism for constructing voronoi diagrams and their generalizations. II. Algorithms for constructing Voronoi diagrams based on the theory of optimal set partitioning // Cybernetics and Systems Analysis, vol. 51, № 4, pp. 489-499 (2015). DOI: 10.1007/s10559-015-9740-y.

4. Aurenhammer F., Klein R., Lee D.-T. Voronoi Diagrams and Delaunay Triangulations. World Scientific Pub Co Inc, 2013. 337 p.

5.Okabe A., Boots B, Sugihara K., Chiu S.N. Spatial Tessellations: Concepts and Applications of Voronoi Diagrams // West Sussex, England: John Wiley and Sons Ltd, second ed., 2000. 696 p.

6.Trubin Stanislav I. Information Space Mapping with Adaptive Multiplicatively Weighted Voronoi Diagrams // Thesis (M.S.) - Origon State University. 2007.

7.Kiseleva E.M., Shor N.Z. Continuous problems of optimal set partitioning: theory, algorithms, applications. Kyiv: Naukova Dumka, 564 p. (2005) [in Russian].

8.Kiseleva E.M., Pritomanova O.M., Zhuravel S.V. Algorithm for Solving a Continuous Problem of Optimal Partitioning with Neurolinguistic Identification of Functions in Target Functional // Journal of Automation and Information Science, vol. 50, № 3, pp. 1-20 (2018). DOI: 10.1615/JAutomatInfScien.v50.i3.10.

9.Shor, N.Z. Nondifferentiable optimization and polynomial problems. Boston; Dordrecht; London: Kluwer Acad. Publ., 412 p. (1998)

10.Stetsyuk P.I. Shor's r-Algorithms: Theory and Practice. In: Optimization Methods and Applications: In Honor of the 80th Birthday of Ivan V. Sergienko. Ed. by Butenko S., Pardalos P.M, Shylo V. Springer. 2017. P. 495-520.

11. Kiseleva E., Hart L., Prytomanova O., Kuzenkov O. An Algorithm to Construct Generalized Voronoi Diagrams with Fuzzy Parameters Based on the Theory of Optimal Partitioning and Neuro-Fuzzy Technologies. URL: http://ceur-ws.org/Vol2386/paper12.pdf.

УДК 519.622.1

ГРНТИ 27.41.1

\title{
ЧИСЛЕННОЕ РЕШЕНИЕ ЗАДАЧИ КОШИ ДЛЯ ОБЫКНОВЕННОГО ДИФФЕРЕНЦИАЛЬНОГО УРАВНЕНИЯ С ПАРАМЕТРОМ ПРИ ПРОИЗВОДНОЙ
}

\author{
Абрамова Вера Викторовна \\ Канд. ф.-м. наук, \\ дочент кафедры автоматизаччи и управления, \\ Набережночелнинский институт (филиал \\ Казанского (Приволжского) федерального университета, \\ Набережные Челны,
}

\footnotetext{
АННОТАЦИЯ

Данная работа посвящена методу коллокации решения обыкновенных дифференциальных уравнений первого порядка с параметром при производной, Основой исследований являются: общая теория приближённых методов анализа и конструктивная теория функций.

ABSTRACT

This paper is devoted to the method of collocation of the solution of first-order ordinary differential equations with the parameter for the derivative. The basis of the research is the general theory of approximate analysis methods and the constructive theory of functions.

Ключевые слова: дифференциальное уравнение, метод коллокации, обратный оператор, сходимость, скорость сходимости, оценка погрешности.
} 